las estrategias (www.HealthyChildren.org/ MediaUsePlan).

\section{REFERENCIAS}

1. Sociedad Argentina de Pediatría. Grupo de Informática. Los pediatras, los padres, los niños e Internet. Buenos Aires: Sociedad Argentina de Pediatría; 2007. [Acceso: 22 de noviembre de 2016]. Disponible en:http:/ / www.sap.org.ar/docs/ ninos_internet.pdf.

2. American Academy of Pediatrics. Council on Communications and Media. Children, adolescents, and the media. Pediatrics 2013;132(5):958-61.

3. American Academy of Pediatrics. Council on Communications and Media. Media use in school-aged children and adolescents. Pediatrics 2016;138(5):e20162592.

4. American Academy of Pediatrics. Council on Communications and Media. Media and young minds. Pediatrics 2016;138(5):e20162591.

5. Kabali HK, Irigoyen MM, Nunez-Davis R, et al. Exposu- re and use of mobile devices by young children. Pediatrics 2015;136(6):1044-50.

6. Christakis DA, Garrison MM, Herrenkohl T, et al. Modifying media content for preschool children: a randomized controlled trial. Pediatrics 2013;131(3):431-8.

7. Radesky JS, Silverstein M, Zuckerman B, et al. Infant selfregulation and early childhood media exposure. Pediatrics 2014;133(5):e1172-8.

8. Reid Chassiakos YL, Radesky J, Christakis D, et al. Children and adolescents and digital media. Pediatrics 2016;138(5):e20162593.

9. American Academy of Pediatrics. Council on Communications and Media. Children, adolescents, obesity, and the media. Pediatrics 2011;128(1):201-8.

10. Holtz $\mathrm{P}$, Appel M. Internetuse and videogaming predict problem behavior in early adolescence. J Adolesc 2011;34(1):4958.

11. Garrison MM, Liekweg K, Christakis DA. Media use and child sleep: the impact of content, timing, and environment. Pediatrics 2011;128(1):29-35.

\title{
Deficiencia de hierro y anemia ferropénica. Guía para su prevención, diagnóstico y tratamiento. Resumen ejecutivo
}

\author{
Iron Deficiency and Iron Deficiency Anemia. Guideline for Prevention, Diagnosis \\ and Treatment. Executive Summary
}

\section{Comité Nacional de Hematología, Oncología y Medicina Transfusional y Comité Nacional de Nutrición}

Texto completo como Suplemento de Archivos Argentinos de Pediatría, en formato electrónico, en su sitio web (véase sección "Suplementos").

\section{INTRODUCCIÓN}

La deficiencia de hierro y la anemia son problemas de salud pública universal por sus consecuencias sobre la salud de los individuos y sobre aspectos sociales y/o económicos, que afectan en distinto grado a todos los países. Ocurre a todas las edades, pero su prevalencia es máxima en niños pequeños y mujeres en edad fértil. Según datos de la Organización Mundial de la Salud (OMS) (2011), más de 2 billones de
Correspondencia: Dr. Hugo Donato, hcdonato@gmail.com

Financiamiento: Ninguno.

Conflicto de intereses: Ninguno que declarar.

Recibido: 4-1-2017 Aceptado: 18-1-2017
Cómo citar: Comité Nacional de Hematología, Oncología y Medicina Transfusional, Comité Nacional de Nutrición. Deficiencia de hierro y anemia ferropénica. Guía para su prevención, diagnóstico y tratamiento. Resumen ejecutivo. Arch Argent Pediatr 2017;115.Supl 4:s68-s82. personas tienen deficiencia de hierro, lo que representa casi el $25 \%$ de la población mundial. La anemia está presente en 800 millones de personas, y son niños 273 millones. Se estima que la sufren, aproximadamente, el 50\% de los niños menores de 5 años y el 25\% de los de 6-12 años de la población mundial. En Argentina, presentan anemia $16 \%$ de los menores de 5 años, $35 \%$ de los niños de 6-24 meses de edad y $20 \%$ de mujeres en edad fértil, con prevalencia variable en distintas regiones, en relación directa con las condiciones socioeconómicas. En los últimos años, en nuestro país, se han tomado medidas de salud pública que han demostrado ser eficaces para disminuir la prevalencia de la deficiencia de hierro y la anemia, pero aún no se ha podido evaluar su impacto.

\section{Coordinadores: Dr. Hugo Donato y Dra. Norma Piazza}

Colaboradores: Dra. Maria C. Rapetti, Dra. Susana de Grandis, Dra. Viviana Bacciedoni, Dra. Marcela Fabeiro, Dra. Alejandra Cedola, Dra. Julieta Hernández, Dr. Marcelo Coirini, Dra. María L. Pita de Portela, Dra. Patricia Sosa y Dra. Virginia Desantadina 
Teniendo en cuenta dicha problemática, esta guía actualizada tiene como finalidad aportar al médico pediatra las pautas y conductas adecuadas para el diagnóstico, la prevención y el tratamiento de la deficiencia de hierro. Las recomendaciones están, en general, basadas en aspectos fisiopatológicos y en opiniones de expertos. No hay otros niveles de evidencia en la bibliografía publicada.

Se detallan a continuación los principales aspectos que se abordan en esta guía.

Definición: Se define anemia como disminución de la masa de glóbulos rojos y/o de la concentración de hemoglobina por debajo del segundo desvío estándar respecto de la media para edad y sexo. Se muestran los valores normales de referencia a distintas edades. Para poblaciones que viven en la altura, se debe calcular que la concentración normal de hemoglobina aumenta en 1,52 g/ dL por cada $1000 \mathrm{~m}$ que se ascienden sobre el nivel del mar (s.n. m.).

Causas: El estado nutricional de hierro de una persona depende del balance determinado por la interacción entre los nutrientes que componen la dieta, la biodisponibilidad, las pérdidas y los requerimientos por crecimiento. La cantidad de hierro que asimila el organismo depende de la cantidad ingerida, la composición de la dieta y la regulación de la absorción por la mucosa intestinal. La biodisponibilidad depende del estado químico en que se encuentra (hemo o no-hemo) y de su interrelación con otros componentes de la dieta, facilitadores o inhibidores de la absorción. El hierro hemo es el de mejor disponibilidad, pues es absorbido sin sufrir modificaciones y sin interrelacionar con otros componentes de la dieta. Por tanto, los alimentos que más hierro aportan son los de origen animal. En las leches, su contenido y biodisponibilidad varía enormemente; la leche materna, con el menor contenido de hierro, presenta la máxima absorción (alrededor del $50 \%$ ). Los niños alimentados a pecho o con fórmulas tienen cubierto su requerimiento diario mínimo, no así los alimentados con leche de vaca no fortificada con hierro. De acuerdo con los valores promedio de requerimientos e ingesta de hierro a distintas edades, hay períodos de la vida en que este balance es negativo, por lo que el organismo debe recurrir al hierro de depósito para sostener una eritropoyesis adecuada. Estos períodos críticos son, sobre todo, tres: el primer año de vida, la adolescencia (en mujeres) y el embarazo. Se enumeran las principales causas de la deficiencia de hierro, que se pueden clasificar, básicamente, en cuatro grandes grupos: a) absorción insuficiente; b) pérdidas aumentadas; c) depósitos disminuidos; d) aumento de requerimientos.

Manifestaciones clínicas: Se enumeran las distintas manifestaciones clínicas de la deficiencia de hierro y se hace hincapié en que se trata de una patología sistémica que, además de producir anemia, puede afectar múltiples órganos. Por su impacto sobre la maduración neurológica del niño, el compromiso del sistema nervioso es la principal manifestación extrahematológica.

Diagnóstico: Se establece la metodología diagnóstica correcta.

a. Interrogatorio detallado: se debe prestar especial atención a aspectos dietéticos, antecedentes perinatales, pérdidas de sangre, trastornos gastrointestinales, hábito de pica, procedencia geográfica y suplemento con hierro recibido.

b. Examen físico completo.

c. Estudios de laboratorio: el hemograma con reticulocitos e índices hematimétricos, la ferremia, la capacidad total de saturación de hierro, el porcentaje de saturación y la ferritina sérica son las pruebas habituales por realizar. Se establecen los valores de corte para cada una de estas determinaciones. Se describe cómo realizar y evaluar la prueba terapéutica.

Diagnóstico diferencial: Se remite a Anemias microcíticas hipocrómicas: guía de diagnóstico diferencial. En nuestro país, las causas más frecuentes de este tipo de anemias son ferropenia, talasemia menor y anemia de la inflamación. Se debe tener en cuenta que algunas de estas patologías pueden coexistir (p. ej., ferropenia y talasemia menor).

Detección: Debido a la alta prevalencia de anemia ferropénica en niños de 6 a 24 meses de edad en nuestro país, se debe pesquisarla mediante la realización sistemática de hemograma y ferritina en el lactante. El estudio se debe realizar entre los 9 y los 12 meses de edad en los recién nacidos de término y entre los 6 y los 9 meses de edad en los prematuros. También se 
recomienda realizar la pesquisa (hemograma, ferremia, capacidad total de saturación de transferrina, porcentaje de saturación y ferritina) en adolescentes mujeres luego de que han presentado la menarca.

Tratamiento: Debe apuntar a corregir la anemia, almacenar hierro en depósitos y corregir la causa primaria. El tratamiento de elección se debe realizar por vía oral, con sulfato ferroso en dosis de 3-6 mg de hierro elemental $/ \mathrm{kg} /$ día. En caso de intolerancia oral grave al sulfato, se puede utilizar hierro polimaltosa. La vía parenteral se debe utilizar solo en casos de intolerancia digestiva grave al hierro oral, patología digestiva que contraindique la vía oral o presunción firme de tratamiento oral insuficiente o inadecuado. Se detalla el procedimiento para administrar el hierro por vía endovenosa. Su indicación debe ser dada por el médico hematólogo. Se establecen pautas para el control del tratamiento y el alta hematológica, y se enfatiza sobre la duración correcta de la administración de hierro y sobre el control de la causa primaria. Se analizan las causas más frecuentes de falla terapéutica: incumplimiento del tratamiento, prescripción inadecuada, falta de resolución de la causa, error diagnóstico, coexistencia con otra patología hematológica y asociación con otras enfermedades. Se destaca la detección de enfermedad celíaca en casos de mala respuesta al tratamiento. Se enumeran las pocas indicaciones de transfusión en la anemia ferropénica.
Profilaxis: Las conductas preventivas que pueden aplicarse están en relación con las condiciones biológicas, ambientales y socioeconómicas de la madre y el niño. Comprenden varios aspectos:

a. Conductas perinatales: Sostén de la adecuación del hierro corporal en la embarazada, incremento del hierro de depósito al nacer.

b. Conductas alimentarias: Se debe promover la lactancia materna exclusiva, favorecer la alimentación complementaria oportuna y adecuada, fortificación de alimentos.

c. Conductas farmacológicas: Se enumeran los grupos de riesgo que deben recibir tratamiento profiláctico y los esquemas terapéuticos para cada uno de ellos. Se puntualiza que no deben recibir suplementos de hierro los niños de término, eutróficos, alimentados con fórmulas regularmente. Se recomienda, siguiendo criterios de la OMS, la desparasitación masiva en zonas de nuestro país con alta prevalencia de uncinariasis.

La asociación de un diagnóstico precoz y certero con la implementación de medidas de prevención adecuadas (tanto a nivel individual como a nivel institucional, regional o nacional) y un tratamiento correcto debería traer como consecuencia una disminución significativa de la prevalencia de deficiencia de hierro en nuestro país y reducir, así, los costos humanos, sociales, educacionales, económicos y laborales que origina esta patología. 